PROCEEDINGS OF THE

AMERICAN MATHEMATICAL SOCIETY

Volume 138, Number 7, July 2010, Pages 2269-2278

S 0002-9939(10)10098-7

Article electronically published on February 23, 2010

\title{
ON THE ENDOFINITENESS OF A KEY MODULE OVER PURE SEMISIMPLE RINGS
}

\author{
NGUYEN VIET DUNG AND JOSÉ LUIS GARCÍA
}

(Communicated by Birge Huisgen-Zimmermann)

\begin{abstract}
Let $R$ be a left pure semisimple ring such that there are no nonzero homomorphisms from preinjective modules to non-preinjective indecomposable modules in $R$-mod, and let $W$ be the left key $R$-module; i.e., $W$ is the direct sum of all non-isomorphic non-preinjective indecomposable direct summands of products of preinjective left $R$-modules. We show that if the module $W$ is endofinite, then $R$ is a ring of finite representation type. This settles a question considered in [L. Angeleri Hügel, A key module over pure-semisimple hereditary rings, J. Algebra 307 (2007), 361-376] for hereditary rings.
\end{abstract}

\section{INTRODUCTION}

A ring $R$ is called left pure semisimple if it has left pure global dimension zero or, equivalently, if every left $R$-module is a direct sum of finitely generated modules. It is well known that left and right pure semisimple rings are precisely the rings of finite representation type, i.e. artinian rings with finitely many isomorphism classes of finitely generated indecomposable left and right modules (see 4, 16, 24]). However it is still an open problem, known as the Pure Semisimplicity Conjecture, whether left pure semisimple rings always have finite representation type (see [19, 27] for historical surveys on the conjecture).

Preinjective modules played an important role in the study of pure semisimple rings (see, e.g., 3, 9, 17, 18, 22, 25, 26, 28]). Inspired by work of Reiten and Ringel 23. on finite-dimensional tame hereditary algebras, Angeleri Hügel [2] recently introduced and studied a key module $W$ over an indecomposable hereditary left pure semisimple $\operatorname{ring} R$. When $R$ is of infinite representation type, this module $W$ is the direct sum of all non-isomorphic non-preinjective indecomposable direct summands of products of preinjective left $R$-modules. It was shown in 2 that, in the above situation, $W$ is a finitely generated product-complete module, and moreover $W$ gives important information on the structure of the ring $R$. For example, no indecomposable direct summand of $W$ can be the source of a left almost split morphism in $R$-mod, and $R$ is of finite representation type provided the endomorphism ring of $W$ has right Morita duality [2, Proposition 4.7, Theorem 4.11]. An interesting

Received by the editors February 5, 2009, and, in revised form, June 17, 2009.

2010 Mathematics Subject Classification. Primary 16G10; Secondary 16D70, 16D90.

Key words and phrases. Pure semisimple ring, ring of finite representation type, preinjective module, endofinite module.

The second author was supported by the Fundación Séneca of the C.A.R.M. 
question, studied in [2, is to determine necessary and sufficient conditions on the ring $R$ for which the key module $W$ is endofinite.

In this paper we give an answer to this question for the wider class of the left pure semisimple rings $R$ satisfying the condition that there are no non-zero homomorphisms from preinjective modules to non-preinjective indecomposable modules in $R$-mod. Our main result shows that the endofiniteness of the key module $W$ is equivalent to $R$ having finite representation type (Theorem 3.6). As a consequence, if such a ring $R$ is not of finite representation type, then $W$ is a finitely generated product-complete non-endofinite left $R$-module whose endomorphism ring is not right artinian (Remark 3.7).

\section{Definitions AND PRELIMINARIES}

Throughout this paper, $R$ is an associative ring with identity. We denote by $R$-mod the category of finitely presented left $R$-modules, and by $R$-Mod the category of all left $R$-modules. The corresponding categories of right $R$-modules are denoted by mod- $R$ and Mod- $R$.

Let $\mathcal{A}$ be a full subcategory of $R$-Mod and $\mathcal{B}$ a full subcategory of $\mathcal{A}$. By Add $\mathcal{B}$ (respectively, add $\mathcal{B}$ ) we denote the class consisting of all modules of $\mathcal{A}$ that are isomorphic to direct summands of (respectively, finite) direct sums of modules in $\mathcal{B}$. If $\mathcal{B}$ consists of a single module $M$, we just write $\operatorname{Add}(M)$ and $\operatorname{add}(M)$ instead. A right $\mathcal{B}$-approximation of a module $M$ of $\mathcal{A}$ is a homomorphism $f: B \rightarrow M$ with $B \in$ add $\mathcal{B}$ such that each map in $\operatorname{Hom}_{R}(X, M)$ with $X \in$ add $\mathcal{B}$ factors through $f$.

Let $\mathcal{C}$ be a family of finitely generated left $R$-modules. By ind $\mathcal{C}$ we mean any family of representatives of the isomorphism classes of indecomposable summands of modules in $\mathcal{C}$. For a ring $R$, we simply write $R$-ind instead of ind $R$-mod. Recall that, for an indecomposable module $M$ in ind $\mathcal{C}$ and $N$ in add $\mathcal{C}$, a homomorphism $f: M \rightarrow N$ is called a left almost split morphism in add $\mathcal{C}$ provided $f$ is not a split monomorphism, and for any module $K$ in add $\mathcal{C}$ and any homomorphism $g: M \rightarrow K$ which is not a split monomorphism, there is a homomorphism $h: N \rightarrow K$ such that $g=h \circ f$. If $f: M \rightarrow N$ is a left almost split morphism in add $\mathcal{C}$, then we say that $M$ is a source of a left almost split morphism in add $\mathcal{C}$. Right almost split morphisms in add $\mathcal{C}$ are defined dually. A non-split exact sequence $0 \rightarrow A \stackrel{f}{\rightarrow} B \stackrel{g}{\rightarrow} C \rightarrow 0$ is called an almost split sequence in add $\mathcal{C}$ if the homomorphisms $f: A \rightarrow B$ and $g: B \rightarrow C$ are, respectively, a left and a right almost split morphism in add $\mathcal{C}$. A homomorphism $f: B \rightarrow C$ is called a right minimal map if for every $g \in \operatorname{Hom}_{R}(B, B), f=f \circ g$ implies that $g$ is an automorphism of $B$.

Recall that $S$ is a ring with enough idempotents if there is a family of pairwise orthogonal idempotents $\left\{e_{\lambda}\right\}_{\lambda \in \Lambda}$ in $S$, so that $S=\bigoplus_{\Lambda} e_{\lambda} S=\bigoplus_{\Lambda} S e_{\lambda}$ (see, e.g., [15]). A left $S$-module will always mean a unitary left $S$-module, and $S$-Mod will denote the category of unitary left $S$-modules. The ring $S$ is left locally coherent if every finitely generated submodule of a finitely presented left $S$-module is finitely presented. A left $S$-module $X$ will be said to be finitely $M$-presented if there is an exact sequence $M^{p} \rightarrow M^{q} \rightarrow X \rightarrow 0$ where $p$ and $q$ are integers. Following [7, 8], a left $S$-module $M$ is called endofinite if $\operatorname{Hom}_{S}(X, M)$ is of finite length over the endomorphism ring of $M$, for any finitely presented left $S$-module $X$. If $R$ is a ring with identity, then a left $R$-module $M$ is endofinite if and only if $M$ is of finite length as a module over its endomorphism ring. A module $M$ is called product-complete 
if $\operatorname{Add}(M)$ is closed under products. It is well known that endofinite modules are product-complete, but the converse is not always true (see [21]).

The following result will be essential in the proof of our main theorem in the next section.

Proposition 2.1. Let $S$ be a left locally coherent ring with enough idempotents, and let ${ }_{S} M$ be a finitely presented injective endofinite left $S$-module. Suppose that every finitely $M$-presented left $S$-module is endofinite and that every finitely presented quotient of ${ }_{S} M$ embeds in a finitely $M$-presented module. Then $M$ has an essential socle.

Proof. See [12, Proposition 2.8].

A cotorsion pair is defined as a pair $(\mathcal{A}, \mathcal{B})$ of classes of modules in $R$-Mod such that $\mathcal{B}=\mathcal{A}^{\perp}$ and $\mathcal{A}={ }^{\perp} \mathcal{B}$, where for any class $\mathcal{E}$ of modules,

$\mathcal{E}^{\perp}=\left\{M \in R\right.$-Mod $\mid \operatorname{Ext}_{R}^{1}(X, M)=0$ for all $\left.X \in \mathcal{E}\right\}$ and

${ }^{\perp} \mathcal{E}=\left\{N \in R\right.$-Mod $\mid \operatorname{Ext} t_{R}^{1}(N, X)=0$ for all $\left.X \in \mathcal{E}\right\}$.

A cotorsion pair $(\mathcal{A}, \mathcal{B})$ is cogenerated by a class $\mathcal{F}$ if $\mathcal{B}=\mathcal{F}^{\perp}$. It is clear that, for any class $\mathcal{F}$ of modules, $\left({ }^{\perp}\left(\mathcal{F}^{\perp}\right), \mathcal{F}^{\perp}\right)$ is a cotorsion pair, cogenerated by $\mathcal{F}$.

We will need the following result, due to Eklof and Trlifaj [14.

Theorem 2.2. Let $R$ be any ring and $(\mathcal{A}, \mathcal{B})$ a cotorsion pair cogenerated by a set of modules. Then for any left $R$-module $L$ there is a short exact sequence $0 \rightarrow K \rightarrow F \rightarrow L \rightarrow 0$ such that $K \in \mathcal{B}$ and $F \in \mathcal{A}$.

Proof. See [14, Theorem 10].

We recall the following definition, following Huisgen-Zimmermann [18] (see also [6]).

Definition 2.3. Let $R$ be a left pure semisimple ring. Then $R$ is left artinian, and there is a strong preinjective partition $R$-ind $=\mathcal{I}_{1} \cup \mathcal{I}_{2} \cup \ldots \cup \mathcal{I}_{n} \cup \ldots \cup \mathcal{I}_{\infty}$, where $\mathcal{I}_{\infty}=\bigcup_{\alpha \geq \omega} \mathcal{I}_{\alpha}$, such that all sets $\mathcal{I}_{\gamma}$ (with $\gamma$ any finite or infinite ordinal) are finite and pairwise disjoint and $\mathcal{I}_{\gamma}$ is a minimal cogenerating set for $\bigcup_{\beta>\gamma} \mathcal{I}_{\beta}$ for each $\gamma$. An indecomposable module $M$ in $R$-mod is called preinjective if $M \in \mathcal{I}_{n}$ for some positive integer $n$. Note that an indecomposable module $M$ lies in $\mathcal{I}_{n}$ if and only if any monomorphism $M \rightarrow N$ with $N \in \operatorname{add}\left(\mathcal{I}_{n} \cup \ldots \cup \mathcal{I}_{\infty}\right)$ splits (see [18, Theorem 4], [9, Lemma 2.1]).

If $R$ is left pure semisimple hereditary, then it is well known that an indecomposable module $M$ in $R$-mod is preinjective if and only if $\operatorname{Hom}_{R}(M, N) \neq 0$ for only finitely many non-isomorphic finitely generated indecomposable left $R$-modules $N$. We now show that, for left pure semisimple rings, this property can be characterized by the condition stated in the abstract of the paper.

Proposition 2.4. Let $R$ be a left pure semisimple ring. The following conditions are equivalent:

(1) For every preinjective left $R$-module $M, \operatorname{Hom}_{R}(M, N) \neq 0$ for only finitely many non-isomorphic indecomposable left $R$-modules $N$.

(2) If $M, N$ are indecomposable left $R$-modules such that $M$ is preinjective and $N$ is not preinjective, then $\operatorname{Hom}_{R}(M, N)=0$.

Proof. (1) $\Rightarrow$ (2) See [13, Lemma 4.10]. 
$(2) \Rightarrow(1)$ Suppose that (2) holds, and assume that $M$ is preinjective in $R$-mod. By Definition 2.3. there is a strong preinjective partition $R$-ind $=\mathcal{I}_{1} \cup \mathcal{I}_{2} \cup \ldots \cup$ $\mathcal{I}_{n} \cup \ldots \cup \mathcal{I}_{\infty}$, and $M \in \mathcal{I}_{n_{0}}$ for some positive integer $n_{0}$. For any $n>n_{0}$, let $M_{n}$ be the intersection of the kernels of all the homomorphisms $M \rightarrow N$, where $N$ is an indecomposable module belonging to some $\mathcal{I}_{k}$ with $k \geq n$.

The sequence $M_{n_{0}+1} \subseteq M_{n_{0}+2} \subseteq \ldots$ is thus an ascending chain of submodules of $M$. Since $M$ is noetherian, there is some $k>n_{0}$ such that $M_{k}=M_{s}$ for all indices $s>k$. We now show that $M_{k}=M$.

Suppose that $M_{k} \neq M$. Then $M / M_{k}$ is a non-zero quotient of $M$; hence by (2) it is a finite direct sum of preinjective modules. Therefore there exists some index $m$ such that all the indecomposable direct summands of $M / M_{k}$ belong to $\mathcal{I}_{1} \cup \ldots \cup \mathcal{I}_{m}$. Now let $s>m, k$. Since $M_{k}=M_{s}$, we have that $M / M_{k}$ is cogenerated by the modules in the sets $\mathcal{I}_{r}(r \geq s)$. Moreover, $M / M_{k}$ is isomorphic to a submodule of a finite direct sum of modules belonging to those sets $\mathcal{I}_{r}$, because $R$ is left artinian. Thus each non-zero indecomposable summand of $M / M_{k}$ is a preinjective module belonging to some $\mathcal{I}_{n}$, with $n \leq m$, which has a monomorphism into a module $N \in$ $\operatorname{add}\left(\mathcal{I}_{n+1} \cup \ldots \cup \mathcal{I}_{\infty}\right)$. But this is a contradiction.

This proves that $M_{k}=M$ for some index $k$. This implies that $\operatorname{Hom}_{R}(M, N)=0$ if $N$ is a preinjective module not belonging to $\mathcal{I}_{1} \cup \ldots \cup \mathcal{I}_{k}$. Since this is a finite set and $\operatorname{Hom}_{R}(M, N)=0$ if $N$ is not preinjective, we see that $\operatorname{Hom}_{R}(M, N) \neq 0$ for only finitely many non-isomorphic indecomposable modules $N$.

Finally, we recall the construction of the right functor ring of a ring $R$, following 15. Let $\left\{U_{\lambda} \mid \lambda \in \Lambda\right\}$ be a complete family of all non-isomorphic finitely presented right $R$-modules, and set $U=\bigoplus_{\lambda \in \Lambda} U_{\lambda}$. Let $S=\left\{f \in H_{R}(U, U) \mid f\left(U_{\lambda}\right)=0\right.$ for almost all $\lambda \in \Lambda\}$. The ring $S$, defined naturally in this way, is called the right functor ring of $R$. Note that $S$ is a left locally coherent ring with enough idempotents, and there is a full and faithful functor $T=U \otimes_{R}-: R$-Mod $\rightarrow S$ Mod, which has a right adjoint. The functor $T$ gives an equivalence between $R$-Mod and the FP-injective left $S$-modules, and $T(M)$ is injective in $S$-Mod if and only if $M$ is pure-injective in $R$-Mod. Moreover, $T$ preserves direct sums and direct products and preserves and reflects finitely presented modules.

We refer the reader to [30, Chapter 10] for more basic properties of the functor ring $S$ and the functor $T$ described above, and to [1, 29, 30] for general properties of rings, modules, and categories, and for all undefined notions used in the text.

\section{MAin RESUlts}

Throughout this section, we assume that $R$ is a left pure semisimple ring that satisfies the following condition:

(A) $\operatorname{Hom}_{R}(X, Y)=0$ for any preinjective left $R$-module $X$ and non-preinjective indecomposable left $R$-module $Y$.

In view of Proposition 2.4 for such a ring $R$, each preinjective left $R$-module has non-zero homomorphisms only to finitely many non-isomorphic indecomposable modules. In particular, left pure semisimple hereditary rings satisfy (A), but not conversely. In fact, non-hereditary rings of finite representation type give obvious examples of left pure semisimple rings that satisfy (A) but are not hereditary.

We start with some notation that will be used throughout this section. Let us denote by $\mathcal{C}_{0}$ the class of all indecomposable left $R$-modules which are not preinjective and by $\mathcal{C}$ the class of all left $R$-modules which have no non-zero preinjective 
direct summands. Let $\mathcal{D}$ be the class of all left $R$-modules that are isomorphic to direct sums of indecomposable direct summands of products of preinjective left $R$-modules. Motivated by the similar construction in [2], we define the left key $R$-module $W$ to be the direct sum of all non-isomorphic non-preinjective indecomposable direct summands of products of preinjective left $R$-modules. Note that when $R$ is an indecomposable hereditary left pure semisimple ring, as in 2], the module $W$ constructed in [2, Theorem 4.3] coincides with our left key module if $R$ is of infinite representation type (see [2, Theorem 4.8]).

We observe also that a module belongs to $\mathcal{D}$ if and only if it is a direct sum of preinjective modules and indecomposable summands of $W$. We first give some preliminary results.

Lemma 3.1. The class $\mathcal{D}$ coincides with $\mathcal{C}^{\perp}$. Consequently, it is closed under extensions.

Proof. Suppose first that $M$ is preinjective and $C$ is a module in $\mathcal{C}$. Let $0 \rightarrow M \stackrel{f}{\rightarrow}$ $X \rightarrow C \rightarrow 0$ be a short exact sequence. Then we write $X=X_{0} \oplus X_{1}$, with $X_{0}$ a direct sum of preinjective modules, and $X_{1} \in \mathcal{C}$. By the condition (A), the image of $f$ is contained in $X_{0}$; hence we may identify $C=X_{1} \oplus L$, where $L$ is the cokernel of the restriction $f: M \rightarrow X_{0}$. But this cokernel is an epimorphic image of $X_{0}$ and belongs to $\mathcal{C}$; hence it is zero by our assumption. Therefore $f$ is a split monomorphism, and this shows that $\operatorname{Ext}_{R}^{1}(C, M)=0$ and $M \in \mathcal{C}^{\perp}$.

Next, note that the class $\mathcal{C}^{\perp}$ is closed under taking direct products and direct summands (see, e.g., [13, Lemma 4.6]), so $\mathcal{D} \subseteq \mathcal{C}^{\perp}$. The converse inclusion follows by combining Proposition 2.4 and the last paragraph of the proof of $[13$, Theorem 4.12].

Lemma 3.2. $W$ is a finitely generated product-complete module, and the indecomposable summands of $W$ are precisely the indecomposable modules in $\mathcal{C}^{\perp} \cap \mathcal{C}$.

Proof. The fact that $W$ is a finitely generated product-complete module was proven in [13, Corollary 4.8]. The second assertion follows from Lemma 3.1.

When $R$ is hereditary and indecomposable, the following result also follows from [2, Proposition 4.6].

Proposition 3.3. Let $N$ be a module in $\mathcal{C}$ which has no direct summand isomorphic to a direct summand of $W$. Then $\operatorname{Hom}_{R}(W, N)=0$.

Proof. Let us first show that there is not any monomorphism $Y \rightarrow N$ if $Y$ is a nonzero indecomposable direct summand of $W$. Assume to the contrary that there is a short exact sequence $0 \rightarrow Y \rightarrow N \stackrel{p}{\rightarrow} Z \rightarrow 0$. We know that $Z=Z_{1} \oplus Z_{0}$ such that $Z_{0}$ belongs to $\mathcal{D}$ and the direct summands of $Z_{1}$ do not belong to $\mathcal{D}$. Let $U=p^{-1}\left(Z_{0}\right)$ so that we get a short exact sequence,

$$
0 \rightarrow Y \rightarrow U \rightarrow Z_{0} \rightarrow 0
$$

and the property that $Y$ and $Z_{0}$ belong to the class $\mathcal{D}$, which is closed under extensions by Lemma 3.1, implies that $U \in \mathcal{D}$. On the other hand, there is also a short exact sequence

$$
0 \rightarrow U \rightarrow N \rightarrow Z_{1} \rightarrow 0 .
$$

Since $U \in \mathcal{D}$ and $Z_{1} \in \mathcal{C}$, we see that the above sequence splits; hence $U$ is isomorphic to a direct summand of $N$. But then $U$ cannot have preinjective direct 
summands since $N \in \mathcal{C}$. Thus any indecomposable direct summand of $U$ would be a direct summand of $W$, which cannot happen by the hypothesis. So we infer that $U=0$, and the monomorphism $Y \rightarrow U$ implies $Y=0$, which is a contradiction.

Suppose now that there is a non-zero homomorphism $f: W \rightarrow N$ and let $X$ be the image of $f$ in $N$. Starting with the short exact sequence $0 \rightarrow K \rightarrow W \rightarrow$ $X \rightarrow 0$, we consider for each module $C \in \mathcal{C}$ the exact sequence $\operatorname{Ext}_{R}^{1}(C, W) \rightarrow$ $\operatorname{Ext}_{R}^{1}(C, X) \rightarrow \operatorname{Ext}_{R}^{2}(C, K)$. Combining Proposition 2.4 with [13, Lemma 4.10], we infer that $C$ has projective dimension at most one, hence $\operatorname{Ext}_{R}^{2}(C, K)=0$. Note also that $\operatorname{Ext}_{R}^{1}(C, W)=0$ by Lemma 3.1. Hence $\operatorname{Ext}_{R}^{1}(C, X)=0$, showing that $X \in \mathcal{D}$ by Lemma 3.1. This implies that all indecomposable direct summands of $X$ are either preinjective or summands of $W$. But if $Y$ is a non-zero indecomposable direct summand of $X$ which is isomorphic to a direct summand of $W$, then we have seen above that there is no monomorphism $Y \rightarrow N$. Thus $X$ would have to be a direct sum of preinjective modules, which contradicts condition (A).

We now consider the cotorsion pair cogenerated by the set of modules $\mathcal{C}_{0}$. Since $\mathcal{D}=\mathcal{C}_{0}^{\perp}$ (because every module in $\mathcal{C}$ is a direct sum of modules in $\mathcal{C}_{0}$ ), this cotorsion pair is $\left({ }^{\perp} \mathcal{D}, \mathcal{D}\right)$. For a left $R$-module $N$ with its endomorphism ring $S$, recall that the local dual of $N$ is defined to be the right $R$-module $D(N)=\operatorname{Hom}_{S}(N, C)$, where $C$ is a minimal injective cogenerator of the category Mod- $S$. The transpose of a finitely presented $R$-module $M$ is denoted by $\operatorname{Tr}(M)$.

Lemma 3.4. A module $X$ belongs to ${ }^{\perp} \mathcal{D}$ if and only if it is a direct sum of a module in $\mathcal{C}$ and a direct sum of indecomposable left $R$-modules that are projective and preinjective.

Proof. The "if" part is clear by Lemma 3.1 .

For the converse, let $X \in{ }^{\perp} \mathcal{D}$ and let $N$ be an indecomposable direct summand of $X$ which does not belong to $\mathcal{C}$. Then $N$ has to be preinjective. Suppose that $N$ is not projective. Then, by Auslander's theorem [5, Theorem I.3.9], there is an almost split sequence in $R$-Mod,

$$
0 \rightarrow A \rightarrow K \rightarrow N \rightarrow 0,
$$

where $A \cong D(\operatorname{Tr}(N))$. Since this sequence is not split, we have that $\operatorname{Ext}_{R}^{1}(N, A) \neq$ 0 . Note that by Proposition 2.4 and [13, Lemma 4.10], we have that $A$ is a preinjective left $R$-module. Hence $A$ belongs to $\mathcal{D}$. But this clearly contradicts the assumption that $N$ is a direct summand of $X \in{ }^{\perp} \mathcal{D}$.

Let $P$ be the direct sum of a set of representatives of the isomorphism classes of indecomposable projective preinjective left $R$-modules. Then $P$ is finitely generated, as the number of isomorphism classes of indecomposable projective modules is finite. Let us set $W_{1}=W \oplus P$. Thus, in view of Lemma 3.2 $W_{1}$ is finitely generated. For hereditary indecomposable rings, a version of the next result is given in [2, Proposition 4.4].

Proposition 3.5. Every finitely presented module of $\mathcal{D}$ is finitely $W_{1}$-presented.

Proof. Let $M \in \mathcal{D}$ be a finitely presented module. We apply Theorem 2.2 to the cotorsion pair $\left({ }^{\perp} \mathcal{D}, \mathcal{D}\right)$. Since it is cogenerated by the set $\mathcal{C}_{0}$ of indecomposable 
(finitely generated) non-preinjective left $R$-modules, we know that there is an exact sequence,

$$
0 \rightarrow K \rightarrow X \rightarrow M \rightarrow 0
$$

with $X \in{ }^{\perp} \mathcal{D}$ and $K \in \mathcal{D}$.

By Lemma 3.4 and the definition of $W_{1}$, we have $X=X_{0} \oplus X_{1}$, where $X_{0} \in$ $\operatorname{Add}\left(W_{1}\right)$ and $X_{1} \in \mathcal{C}$ has no direct summands in $\mathcal{D}$. Since $K \in \mathcal{D}$ and there are no non-zero homomorphisms from modules in $\mathcal{D}$ to $X_{1}$ by Proposition 3.3 , we have that the image of $K$ is inside $X_{0}$. Thus $X_{1}$ is isomorphic to a direct summand of $M$. If $X_{1} \neq 0$, then $X_{1}$ would have a direct summand in $\mathcal{D}$, which is contrary to our assumptions. Hence $X_{1}=0$, and $X=X_{0}$ belongs to $\operatorname{Add}\left(W_{1}\right)$.

In particular, for every finitely presented module $N \in \mathcal{D}$ there is an epimorphism $Z \rightarrow N$ where $Z$ is a finitely presented module of $\operatorname{Add}\left(W_{1}\right)$; hence $Z \in \operatorname{add}\left(W_{1}\right)$.

Now, let $X=\bigoplus_{i \in I} X_{i}$ and $K=\bigoplus_{j \in J} K_{j}$ be indecomposable decompositions, and set $K_{F}=\bigoplus_{j \in F} K_{j}$ for each finite subset $F \subseteq J$. Since $\mathcal{D}$ is closed under direct summands and finite direct sums, we have $K_{F} \in \mathcal{D}$. As $K$ is the direct union of its submodules $K_{F}$, we have that $M \cong X / K \cong \lim \left(X / K_{F}\right)$ (see [29, Proposition IV.8.9]).

Since $M$ is finitely presented, the functor $\operatorname{Hom}_{R}(M,-)$ commutes with direct limits, and hence $M$ is isomorphic to a direct summand of $X / K_{F}$ for some $F$. But then $M$ is isomorphic to a direct summand of a module of the form $\left(\bigoplus_{i \in F_{1}} X_{i}\right) / K_{F}$, where $F_{1}$ is a finite subset of $I$, because $M$ is finitely generated. Therefore, we conclude that there is a left $R$-module $N$ so that $M \oplus N \cong Y / L$, where $Y, L$ are finitely generated and $Y \in \operatorname{add}\left(W_{1}\right), L \in \mathcal{D}$.

We may thus suppose that $N \cong Y_{1} / L$ for some submodule $Y_{1} \subseteq Y$, so that $M \cong Y / Y_{1}$. By Lemma 3.1. $\mathcal{D}$ is closed under extensions, and $N$, being a quotient of $Y$, is in $\mathcal{D}$ by Proposition 3.3. Hence we have that $Y_{1} \in \mathcal{D}$. So we get a short exact sequence $0 \rightarrow Y_{1} \rightarrow Y \rightarrow M \rightarrow 0$, with $Y \in \operatorname{add}\left(W_{1}\right)$ and $Y_{1}$ a finitely presented module of $\mathcal{D}$. By the above observation, there is an epimorphism $Z \rightarrow Y_{1}$ with $Z \in \operatorname{add}\left(W_{1}\right)$. Then the right exact sequence $Z \rightarrow Y \rightarrow M \rightarrow 0$ shows that $M$ is finitely $W_{1}$-presented.

We are now ready to prove our main result.

Theorem 3.6. Let $R$ be a left pure semisimple ring such that there are no nonzero homomorphisms from preinjective modules to non-preinjective indecomposable modules in $R$-mod. The left key $R$-module $W$ is endofinite if and only if $R$ is of finite representation type.

Proof. If $R$ is of finite representation type, then it is well known that every left (or right) $R$-module is endofinite (see [20, Theorem 6]).

For the converse, suppose that $W$ is endofinite. If $W=0$, this would mean that any direct product of preinjective modules in $R$-mod is a direct sum of preinjective modules. Therefore, $R$ is of finite representation type by [18, Corollary B] (cf. also [13, Corollary 3.12]).

Thus, we now assume that $W$ is non-zero. We will get a contradiction by following several steps. Let $S$ be the right functor ring of $R$. We know that $S$ is left locally noetherian [30,53.7] and that there is a full and faithful functor $T: R$-Mod $\rightarrow S$-Mod such that $T(M)$ is injective as a left $S$-module for every module $M$ in $R$-Mod. Let $W_{1}$ be the module as in Proposition 3.5

(1) $W_{1}$ is a finitely presented endofinite module. 
We know that a finite direct sum of endofinite modules is endofinite [7, Proposition 4.3]. Since $W$ is endofinite and each preinjective left $R$-module is endofinite [13. Corollary 3.6], we see that $W_{1}$ is endofinite.

(2) A left $S$-module $L$ is finitely $T\left(W_{1}\right)$-presented if and only if there is a finitely $W_{1}$-presented module $N$ such that $T(N) \cong L$.

If $N$ is finitely $W_{1}$-presented, then there is an exact sequence

$$
W_{1}^{r} \rightarrow W_{1}^{t} \rightarrow N \rightarrow 0
$$

Since the functor $T$ is additive and right exact, we get the corresponding exact sequence in $S$-Mod,

$$
T\left(W_{1}\right)^{r} \rightarrow T\left(W_{1}\right)^{t} \rightarrow T(N) \rightarrow 0,
$$

which shows that $T(N)$ is finitely $T\left(W_{1}\right)$-presented.

Conversely, let there be given an exact sequence in $S$-Mod,

$$
T\left(W_{1}\right)^{r} \stackrel{f}{\rightarrow} T\left(W_{1}\right)^{s} \rightarrow L \rightarrow 0 .
$$

Since $T$ is an additive full functor, we have that there is a homomorphism $a$ : $W_{1}^{r} \rightarrow W_{1}^{s}$ such that $T(a)=f$. This gives rise to an exact sequence in $R$-Mod $W_{1}^{r} \stackrel{a}{\rightarrow} W_{1}^{s} \rightarrow Y \rightarrow 0$. Then $Y \cong \operatorname{Coker}(a)$ so that $T(Y) \cong \operatorname{Coker}(T(a)) \cong$ $\operatorname{Coker}(f) \cong L$, because $T$ is right exact. Thus $L \cong T(Y)$, with $Y$ being a finitely $W_{1}$-presented module.

(3) If $L$ is a finitely $T\left(W_{1}\right)$-presented left $S$-module, then $L$ is endofinite.

By (2) above, $L \cong T(M)$ for some $M$ which is finitely $W_{1}$-presented. Then $M \in \mathcal{D}$ by Proposition 3.3 and assumption (A). Therefore $M$ is a finite direct sum of preinjective modules and direct summands of $W$, and hence $M$ is endofinite, as shown in (1). Then $T(M)$ is also endofinite, by [8, 3.6, Lemma 1].

(4) Let $q: T\left(W_{1}\right) \rightarrow L$ be an epimorphism of $S$-Mod. Then $L$ embeds in a finitely $T\left(W_{1}\right)$-presented left $S$-module.

We know that $L$ is finitely generated and hence finitely presented because $S$ is left locally noetherian. Thus $L$ embeds in a module of the form $T(N)$ for $N$ a finitely presented left $R$-module by [8, 3.3, Lemma 3] (cf. [10, Theorem 2.4]).

We then construct the homomorphism $f: T\left(W_{1}\right) \rightarrow T(N)$ by composing the epimorphism $q$ with the canonical inclusion. Since $T$ is full, we know that there is a homomorphism $a: W_{1} \rightarrow N$ such that $T(a)=f$.

By Proposition 3.3 and condition (A), there are no non-zero homomorphisms from $W_{1}$ to any indecomposable module which does not belong to $\mathcal{D}$. Therefore there is a direct summand $N_{0} \subseteq N$ such that $\operatorname{Im}(a) \subseteq N_{0}$ and $N_{0} \in \mathcal{D}$. Thus $a: W_{1} \rightarrow N$ factors through the inclusion $N_{0} \hookrightarrow N$.

Consequently, $f=T(a)$ can be factored through the direct summand $T\left(N_{0}\right)$ of $T(N)$, and therefore the image $L$ of $T(a)$ is contained in $T\left(N_{0}\right)$. Since $N_{0} \in \mathcal{D}$, the module $N_{0}$ is finitely $W_{1}$-presented by Proposition 3.5. Then $T\left(N_{0}\right)$ is finitely $T\left(W_{1}\right)$-presented by $(2)$, and this proves (4).

(5) Each indecomposable direct summand of $W$ is the source of a left almost split morphism in R-mod.

We use Proposition 2.1, applied to the left locally coherent ring $S$ and to the injective left $S$-module $T\left(W_{1}\right)$. We know that the hypotheses of the proposition are satisfied by points (1), (3) and (4). The conclusion is that $T\left(W_{1}\right)$ has an essential socle. Therefore, if $M$ is any indecomposable direct summand of $W$, then $T(M)$ 
has an essential socle, which implies that $M$ is the source of a left almost split morphism in $R$-mod, by [11, Lemma 2.4].

Finally, we have reached the contradiction, because $W \neq 0$ and we know that each indecomposable direct summand of $W$ is not the source of a left almost split morphism in $R$-mod, by [13, Corollary 4.8].

We conclude the paper with the following.

Remark 3.7. (a) Let $R$ be a two-sided artinian hereditary indecomposable left pure semisimple ring. Angeleri Hügel considered in [2, Section 5] the cotorsion pair $(\mathcal{M}, \mathcal{L})$ cogenerated by the preprojective component $\mathbf{p}$ in $\operatorname{Mod}-R$, and she showed that the key module $W$ in $R$-Mod is endofinite if and only if the class $\mathcal{M}$ is closed under direct limits in Mod- $R$ (see [2, Theorem 5.6]). Combining this result with our Theorem 3.6. we deduce that the class $\mathcal{M}$ is closed under direct limits precisely when $R$ is of finite representation type.

(b) Let $R$ and $W$ be as in Theorem 3.6 and suppose $R$ is not of finite representation type. Then, by combining Theorem 3.6 with Proposition 2.4 and [13, Theorem 4.12], we obtain a complete description of the module $W$ as follows: $W$ is a finitely generated product-complete non-endofinite left $R$-module, and the endomorphism ring $S$ of $W$ is not right artinian, because $W$ is finitely presented over its endomorphism ring [21, Proposition 3.9]. Moreover, if $f_{k}: M_{k} \rightarrow I_{k}$ are the minimal right $\mathcal{C}$-approximations of all the indecomposable injective left $R$-modules, then the indecomposable summands of $W$ are precisely the indecomposable summands of $M_{k}$ and of the kernels $\operatorname{Ker}\left(f_{k}\right)$.

\section{REFERENCES}

[1] F. W. Anderson and K. R. Fuller, "Rings and Categories of Modules", Springer-Verlag, New York, 1992. MR1245487(94i:16001)

[2] L. Angeleri Hügel, A key module over pure-semisimple hereditary rings, J. Algebra 307 (2007), 361-376. MR 2278060 (2007j:16021)

[3] L. Angeleri Hügel and H. Valenta, A duality result for almost split sequences, Colloq. Math. 80 (1999), 267-292. MR1703897 (2000h:16025)

[4] M. Auslander, Representation theory of Artin algebras. II, Comm. Algebra 1 (1974), 269-310. MR0349747 (50:2240)

[5] M. Auslander, Functors and morphisms determined by objects, Lecture Notes in Pure and Appl. Math., 37, Dekker, New York, 1978, 1-244. MR0480688 (58:844)

[6] M. Auslander and S. O. Smalø, Preprojective modules over Artin algebras, J. Algebra 66 (1980), 61-122. MR.591246 (83a:16039)

[7] W. W. Crawley-Boevey, Modules of finite length over their endomorphism rings, in "Representations of algebras and related topics" (Eds. S. Brenner and H. Tachikawa), London Math. Soc. Lecture Note Series, Vol. 168, Cambridge University Press, 1992, pp. 127-184. MR:1211479 (94h:16018)

[8] W. W. Crawley-Boevey, Locally finitely presented additive categories, Comm. Algebra 22 (1994), 1641-1674. MR1264733 (95h:18009)

[9] N. V. Dung, Strong preinjective partitions and almost split morphisms, J. Pure Appl. Algebra 158 (2001), 131-150. MR1822837 (2002c:16019)

[10] N. V. Dung and J. L. García, Additive categories of locally finite representation type, J. Algebra 238 (2001), 200-238. MR.1822190(2002f:18021)

[11] N. V. Dung and J. L. García, Copure semisimple categories and almost split maps, J. Pure Appl. Algebra 188 (2004), 73-94. MR2030807 (2004m:18009)

[12] N. V. Dung and J. L. García, Endofinite modules and pure semisimple rings, J. Algebra 289 (2005), 574-593. MR2142386 (2006h:16036)

[13] N. V. Dung and J. L. García, Preinjective modules over pure semisimple rings, J. Pure Appl. Algebra 212 (2008), 1207-1221. MR2387596 (2009b:16031) 
[14] P. Eklof and J. Trlifaj, How to make Ext vanish, Bull. London Math. Soc. 33 (2001), 41-51. MR:1798574 (2001i:16015)

[15] K. R. Fuller, On rings whose left modules are direct sums of finitely generated modules, Proc. Amer. Math. Soc. 54 (1976), 39-44. MR0393133 (52:13943)

[16] K. R. Fuller and I. Reiten, Note on rings of finite representation type and decompositions of modules, Proc. Amer. Math. Soc. 50 (1975), 92-94. MR0376768 (51:12943)

[17] I. Herzog, A test for finite representation type, J. Pure Appl. Algebra 95 (1994), 151-182. MR1293051 (95h:16011)

[18] B. Huisgen-Zimmermann, Strong preinjective partitions and representation type of Artinian rings, Proc. Amer. Math. Soc. 109 (1990), 309-322. MR.1007520 (90i:16026)

[19] B. Huisgen-Zimmermann, Purity, algebraic compactness, direct sum decompositions, and representation type, in "Infinite Length Modules" (Eds. H. Krause and C. M. Ringel), Trends Math., Birkhäuser, Basel, 2000, pp. 331-367. MR1789225 (2002c:16008)

[20] B. Huisgen-Zimmermann and W. Zimmermann, On the sparsity of representations of rings of pure global dimension zero, Trans. Amer. Math. Soc. 320 (1990), 695-711. MR965304 (90k:16036)

[21] H. Krause and M. Saorín, On minimal approximations of modules, in "Trends in the Representation Theory of Finite-Dimensional Algebras" (Eds. E. L. Green and B. HuisgenZimmermann), Contemp. Math., 229, Amer. Math. Soc., Providence, RI, 1998, pp. 227-236. MR:1676223 (99m:16002)

[22] F. Okoh, Direct sum decomposition of the product of preinjective modules over right pure semisimple hereditary rings, Comm. Algebra 30 (2002), 3037-3043. MR.1908256 (2003d:16015)

[23] I. Reiten and C. M. Ringel, Infinite dimensional representations of canonical algebras, Canad. J. Math 58 (2006), 180-224. MR2195596(2006k:16006)

[24] C. M. Ringel and H. Tachikawa, QF-3 rings, J. Reine Angew. Math. 272 (1974), 49-72. MR0379578 (52:483)

[25] D. Simson, Partial Coxeter functors and right pure semisimple hereditary rings, J. Algebra 71 (1981), 195-218. MR627434 (82m:16031)

[26] D. Simson, On right pure semisimple hereditary rings and an Artin problem, J. Pure Appl. Algebra 104 (1995), 313-332. MR1361578 (96m:16018)

[27] D. Simson, A class of potential counterexamples to the pure semisimplicity conjecture, in "Advances in Algebra and Model Theory" (Eds. M. Droste and R. Göbel), Algebra, Logic and Applications, Gordon and Breach, Amsterdam, 1997, pp. 345-373. MR.1683552 (2000d:16019)

[28] D. Simson, An Artin problem for division ring extensions and the pure semisimplicity conjecture. II, J. Algebra 227 (2000), 670-705. MR1759841(2002c:16025)

[29] B. Stenström, "Rings of Quotients", Springer-Verlag, New York-Heidelberg, 1975. MR.0389953 (52:10782)

[30] R. Wisbauer, "Foundations of Module and Ring Theory", Algebra, Logic and Applications, Vol. 3, Gordon and Breach, Philadelphia, PA, 1991. MR.1144522 (92i:16001)

Department of Mathematics, Ohio University - Zanesville, Zanesville, Оhio 43701

E-mail address: nguyend2@ohiou.edu

Department of Mathematics, University of Murcia, 30100 Murcia, Spain

E-mail address: jlgarcia@um.es 